\title{
Robotically Controlled Slosh-Free Motion of an Open Container of Liquid
}

\author{
John Feddema, Clark Dohrmann, Gordon Parker, \\ Rush Robinett, Vicente Romero, Dan Schmitt ${ }^{\dagger}$ \\ Sandia National Laboratories \\ Albuquerque, NM 87185
}

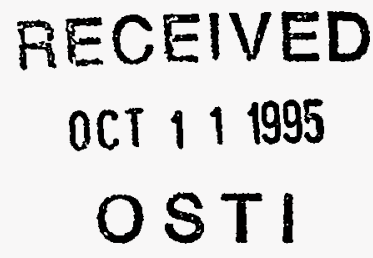

dynamics of a liquid booster can alter the rocket trajectory [1]. This same technology could be used in robotic applications for controlling open containers of liquids in a high speed process.

This paper describes two methods for controlling the surface of a liquid in an open container as it is being carried by a robot arm. Both methods make use of the fundamental mode of oscillation and damping of the liquid in the container as predicted from a boundary element model of the fluid. The first method uses an infinite impulse response filter to alter an acceleration profile so that the liquid remains level except for a single wave at the beginning and end of the motion. The motion of the liquid is similar to that of a simple pendulum. The second method removes the remaining two surface oscillations by tilting the container parallel to the beginning and ending wave. A double pendulum model is used to determine the trajectory for this motion. Experimental results of a FANUC S-800 robot moving a $230 \mathrm{~mm}$ diameter hemispherical container of water are presented.

\section{Introduction}

There are many industrial processes where a rapid, controlled slosh-free movement of an open container of liquid would be beneficial. For example, in the steel industry it is desirable to move molten metal from the melting furnace to the forming stations as quickly as possible without spilling the molten steel. Other applications include the transport of molten glass, melted plastic, hot tar, and hazardous chemicals.

Most previous work in the modeling and control of moving liquid filled containers has been performed for rocket applications where the fluid
This paper presents two methods for the robotic delivery of an open container of liquid without residual end point sloshing. The first method plans a translational motion profile so that the liquid pitches only at the beginning and end of the motion. The second method tilts the container so that the normal of the liquid is maintained opposite to the resultant acceleration of the motion and gravity.

The next section discusses the modeling of the liquid and how this model is used in an open loop control scheme. The following two sections describe the controlled horizontal motion and slosh-free motion control methods. The final two sections present experimental results and conclusions.

\section{Modeling}

The objective of this section is to provide an approximate two dimensional model of a fluid in an open container which can be used to estimate the surface wave oscillation frequencies. These frequencies are used to determine the open loop control parameters for slosh-free motion as discussed in the following two sections.

Throughout this discussion, we will consider the container shown in Figure 1. To model the motion of the fluid, momentum conservation must be applied. Assuming the fluid to be irrotational, the velocity potential $\phi(x, y, t)$

$\dagger$ This work performed at Sandia National Laboratories supported by the U.S. Department of Energy under contract DE-AC0494AL85000. 
governs the flow field such that it's gradient represents the fluid velocity at any point:

$$
\underline{v}_{\text {fluid }}=\nabla \phi(x, y, t)
$$

Assuming incompressibility, conservation of mass requires that the divergence of the velocity be zero everywhere within the fluid. This requirement implies that $\phi(x, y, t)$ satisfy Laplace's equation in the domain:

$$
\nabla \bullet \underline{v}_{\text {fluid }}=0 \Rightarrow \nabla^{2} \phi(x, y ; t)=0
$$

Under the additional assumption of negligible viscous effects (which completes the set of assumptions characterizing potential flow), the time-consistency of the problem is established by Kelvin's theorem [2], which states that an inviscid and initially irrotational flow will always remain irrotational, and therefore that Laplace's equation will apply throughout time.

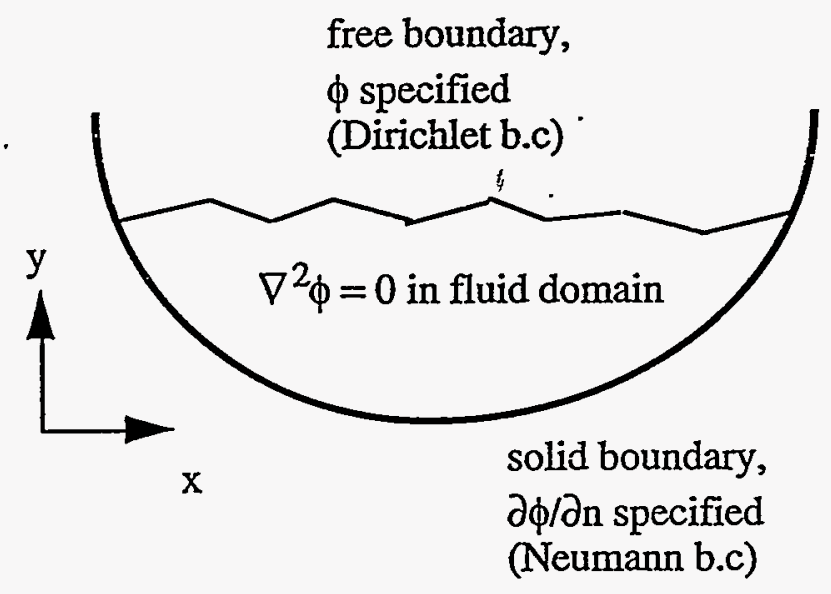

Figure 1. Modeled boundary conditions.

We may fully characterize the flow over the time span of interest by resolving the time-dependent velocity field within the domain. At any point in time, the solution of Laplace's equation for the velocity potential $\phi(x, y, t)$ with subsequent use of Equation (1) yields the velocity field. The boundary conditions for the Laplace part of our problem are illustrated in Figure 1. Note that nonlinearity and time dependence are introduced through the boundary conditions to the Laplace problem and through the time-changing geometry of the domain due to the movement of the free surface and of other boundaries (such as the container boundaries) whose motion may be prescribed as a function of time.

A boundary element method (BEM) has been implemented to solve Equations (1) and (2) for arbitrarily shaped containers experiencing arbitrary combinations of horizontal, vertical, and angular pitching motions in the modeling plane. From analytical comparison to previous codes and limited experimental testing, the model appears to predict sloshing frequency quite well for any type of container motion or geometry. For more detail on this BEM, see [3].

The next two sections will use the fundamental mode of oscillation as predicted by this BEM to generate a controlled input to the system which will result in a slosh-free motion. $=$

\section{Controlled Horizontal Motion}

In this section, the motion of the container is restricted to simply translating the liquid along the $\mathrm{x}$ axis as shown in Figure 2. Using the BEM results for the first mode, the dynamics of the fluid relative to the container are assumed to be equivalent to an underdamped second order system:

$$
\ddot{\theta}+2 \zeta \omega \dot{\theta}+\omega^{2} \theta=\frac{1}{r_{m}} \ddot{x}
$$

where $\theta$ is the angle of the liquid with respect to the $x$ axis, $\zeta$ is the damping ratio, $\omega$ is the natural frequency of the liquid within the container, $r_{m}$ is the virtual length of the center of mass of the liquid to the center of the hemispherical container, and $\ddot{x}$ is the acceleration of the container.

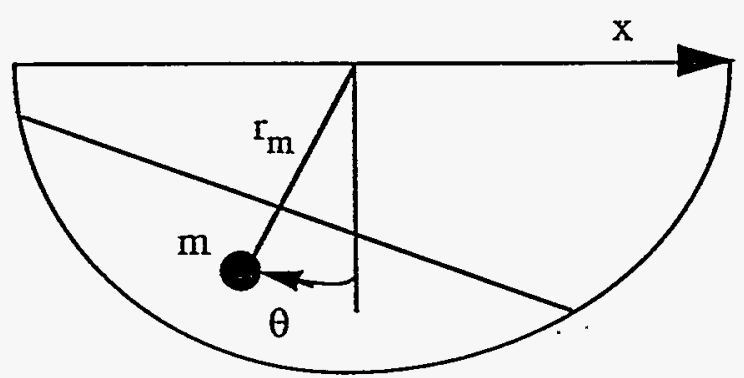

Figure 2. Simple horizontal model of fundamental mode of oscillation. 
There are several ways to dampen an oscillatory system [4][5][6]. In this paper, an infinite impulse response (URR) filter is used to pre-shape the input $\ddot{x}$ of an underdamped second order system so that the overall system becomes a critically damped third order plant. The IIR filter is implemented via the following difference equation [6]:

$$
\begin{aligned}
y(k)= & -a_{1} y(k-1)-a_{2} y(k-2)-a_{3} y(k-3) \\
& +b_{0} u(k)+b_{1} u(k-1)+b_{2} u(k-2) \\
& +b_{3} u(k-3)+b_{4} u(k-4)
\end{aligned}
$$

where $y(k)$ is the output of the filter and $u(k)$ is the input to the filter $(\ddot{x})$ at discrete time $\mathrm{k}$. The IIR filter coefficients $a_{i}$ and $b_{i}$ are a function of the natural frequency $\omega$, damping ratio $\zeta$, a scaling factor $\kappa$, and the sampling period $T$. The scaling factor $\kappa$ is used to shorten the settling time of the shaping filter. The larger the value, the shorter the settling time of the filter.

However, a shorter settling time means the filter may try to drive the system faster than capable.

Typically, $\mathrm{K}$ is chosen so that the settling time is approximateiy equal to one half the period of oscillation.

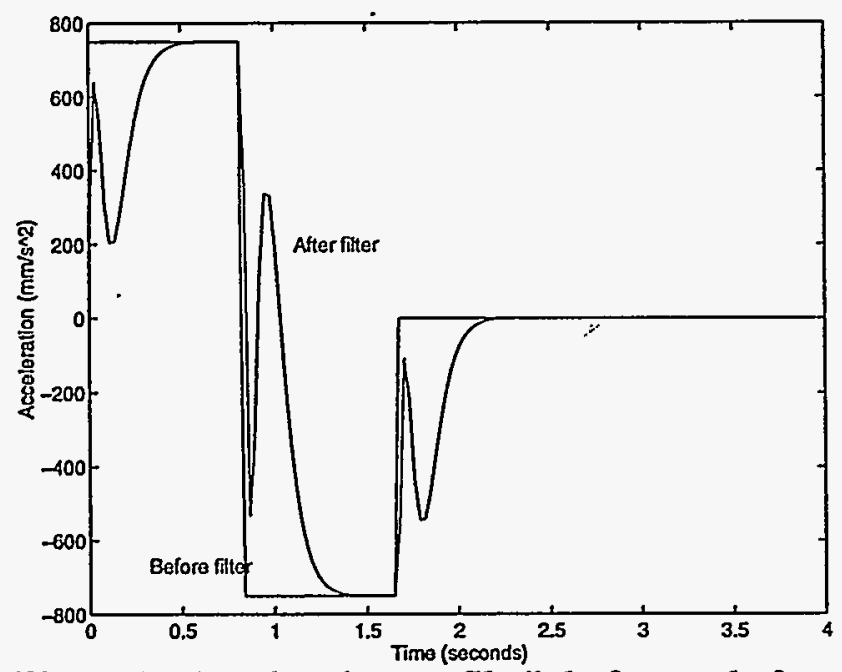

Figure 3. Acceleration profile $\ddot{x}$ before and after filtering.

Figure 3 shows the acceleration profile along the $x$ axis before and after filtering. Notice that the filter delays the profile. The natural frequency and damping ratio used in the figure are the values determined in the Experimentation Section below. Figure 4 shows the estimate angle $\theta$ (from Equation (3)) before and after filtering. As seen in the Figure, this method eliminates residual oscillation at the end of the motion.

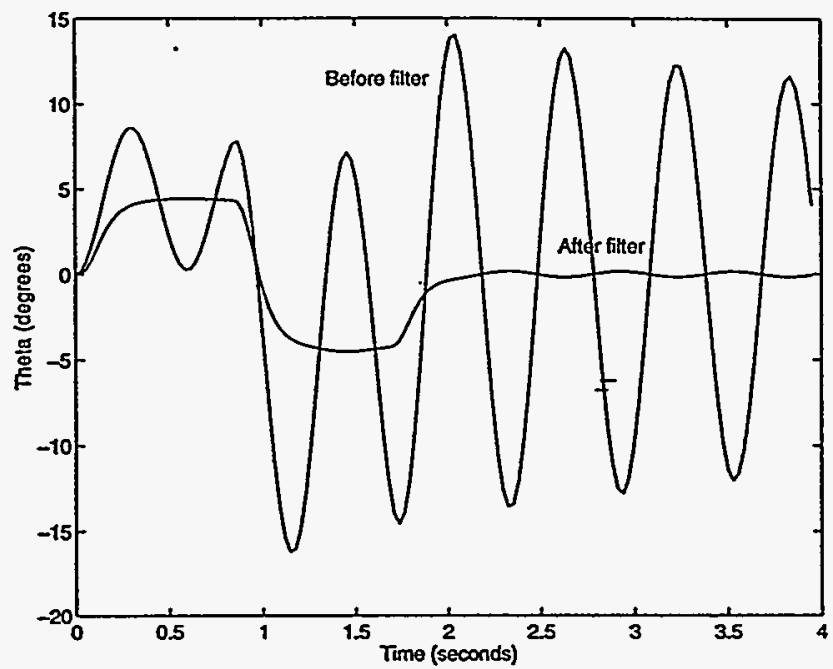

Figure 4. The predicted angle $\theta$ of the fluid relative to the container.

\section{Slosh-Free Motion}

Some applications require that the liquid not move relative to the container. For example, in the steel industry it may be desirable to keep the molten metal from moving up the sides of the container wall where a thin film will form. In order to do this, the robot must be able to rotate about an axis that is orthogonal to the plane of translation.

Similar to the previous section, a simplified model assumes that the liquid surface remains flat during the robotic maneuvers and, therefore, can be modeled as an oscillating pendulum. Adding the rotation of the container, the liquid and container can be modeled by a double pendulum with a moving base as shown in Figure 5 . The symbol $m$ is the mass of the liquid, $r_{m}$ is the length of the equivalent pendulum, $r_{L}$ is the distance from the center of rotation of the robot to the center of rotation of the pendulum, $x$ is the translational position of the center of rotation of the robot, and $\theta$ and $\beta$ are the angle of rotation of the pendulum and the robot, respectively. 


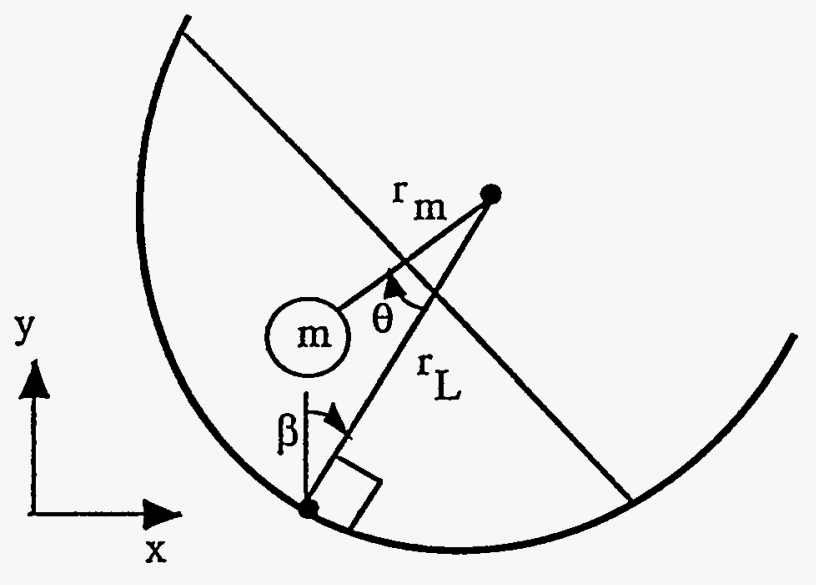

Figure 5 - Double pendulum with a moving base.

The equations of motion for the moving base, double pendulum are derived with Lagrange's equations. The position of the center of mass of the liquid is

$$
\begin{gathered}
x_{m}=x+\dot{r}_{L} \sin \beta-r_{m} \sin (\beta+\theta) \\
y_{m}=r_{L} \cos \beta-r_{m_{i}} \cos (\beta+\theta) \\
\underline{r}_{m}=x_{m} \underline{\hat{i}}+y_{m} \underline{\hat{j}}
\end{gathered}
$$

and the potential and kinetic energy functions are

and

$$
V=m g y_{m}
$$

$$
T=\frac{1}{2} m \underline{r}_{m} \cdot \dot{\underline{r}}_{m}
$$

which produce the Lagrangian $L=T-V$.

A Rayleigh dissipation function is employed to account for the damping force between the container and the liquid

$$
\Re=\frac{1}{2} c_{m} \dot{\theta}^{2}
$$

where $c_{m}$ is the Rayleigh damping coefficient. The equation of motion of interest is the Lagrange equation with respect to $\theta$ which is derived from

$$
\frac{d}{d t}\left(\frac{\partial L}{\partial \dot{\theta}}\right)-\frac{\partial L}{\partial \theta}+\frac{\partial \Re}{\partial \dot{\theta}}=0
$$

and results in the governing equation of motion:

$$
\begin{aligned}
& \ddot{\theta}+\left[1-\frac{r_{L}}{r_{m}} \cos \theta\right] \ddot{\beta}+\frac{g}{r_{m}} \sin (\theta+\beta)-\frac{r_{L}}{r_{m}} \dot{\beta}^{2} \sin \theta \\
& -\frac{\ddot{x}}{r_{m}} \cos (\theta+\beta)+\frac{c_{m}}{m r_{m}^{2}} \dot{\theta}=0
\end{aligned}
$$

Setting $\beta, \dot{\beta}, \ddot{\beta}$ equal to zero and assuming a small angle for $\theta$, this equation results in the same linear, damped, harmonic oscillator model used in the previous section. As will be seen in the next section, estimates of the natural frequency and damping ratio can be determined from the BEM or experimentally from smallangle, free vibration data using standard techniques.

The goal of slosh-free motion is to have no relative motion between the container and the fluid. With reference to Figure 5 , this is equivalent to keeping the angle $\theta$ equal to zero for all times. Setting $\theta, \dot{\theta}, \ddot{\theta}$ equal to zero in Equation (12), one obtains

where

$$
\ddot{\beta}+\omega_{o}^{2} \sin (\beta)=\frac{\omega_{o}^{2}}{g} \ddot{\ddot{x}} \cos (\beta)
$$

$$
\omega_{o}^{2}=\frac{g}{r_{m}-r_{L}} .
$$

Slosh-free motion is accomplished by controlling the angle $\beta$ so that Equation (13) is satisfied. From equation (14), notice that Equation (13) will become unstable if $r_{m}$ is less than $r_{L}$. Fortunately, with a $6 \mathrm{DOF}$ robot, we can make the center of rotation of the container in Figure 5 be such that $r_{L}=0$.

Of particular interest to this study are constant acceleration maneuvers in which

$$
\ddot{x}=\varepsilon g
$$

where the magnitude of the scalar $\varepsilon$ is typically less than unity. The analytical solution to (13) 
developed below is based on a modification of a perturbation procedure presented in [7]. The basic approach is to expand $\beta$ in ascending powers of $\varepsilon$ as

$$
\beta(t, \varepsilon)=\varepsilon \beta_{o}(\omega t)+\varepsilon^{3} \beta_{2}(\omega t)+\varepsilon^{5} \beta_{4}(\omega t)+\ldots
$$

where $\omega$ is expanded in ascending powers of $\varepsilon$ as

$$
\omega=\omega_{o}+\varepsilon^{2} \omega_{2}+\varepsilon^{4} \omega_{4}+\ldots
$$

Substituting Equations (15-17) into Equation (13), equating coefficients of like powers of $\varepsilon$, and solving the resulting differential equations such that there are no secular terms and the initial conditions $\beta(0)=0$ and $\dot{\beta}(0)=0$ are satisfied yields

$$
\begin{aligned}
& \beta(t, \varepsilon)=\varepsilon(1-\cos \omega t)+\frac{\varepsilon^{3}}{192}(-64+63 \cos \omega t \\
& +\cos 3 \omega t)+\frac{\varepsilon^{5}}{61440}(12288-11985 \cos \omega t \\
& -300 \cos 3 \omega t-3 \cos 5 \omega t)+\ldots
\end{aligned}
$$

where

$$
\omega=\left(1+\frac{3 \varepsilon^{2}}{16}-\frac{69 \varepsilon^{4}}{1024}+. .\right) \omega_{o}
$$

Using the same parameters as determined in the Experimentation Section below, a time history of $\beta$ for a square wave acceleration is shown in Figure 6. Equation (19) was used to compute $\omega$, and Equation (18) was used to compute $\beta$. With this acceleration profile, fluid will be sloshfree if the time of acceleration is a multiple of the period of oscillation.

$$
t_{a c c}=\frac{2 \pi n}{\omega}
$$

where $n$ is an integer.

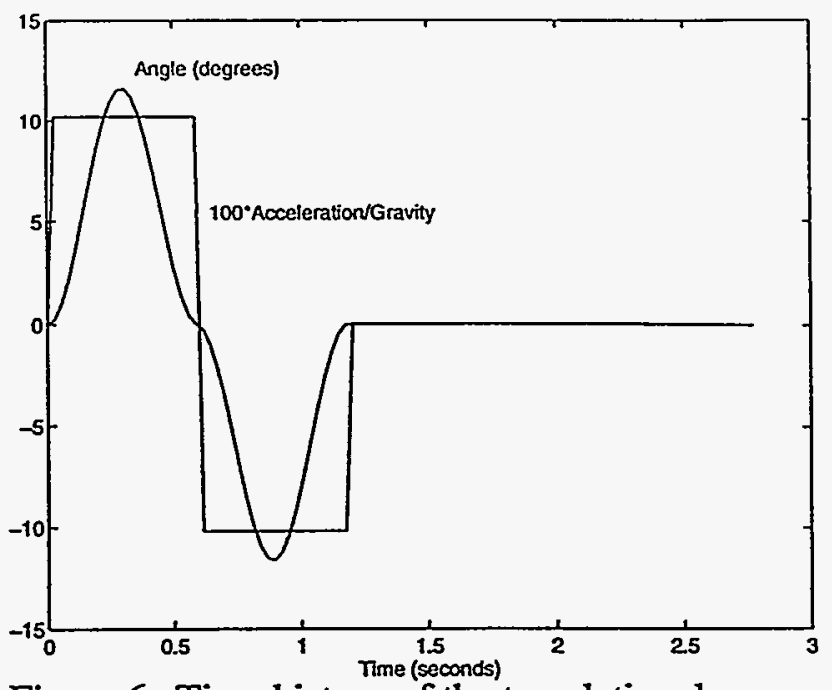

Figure 6. Time history of the translational acceleration $\ddot{x}$ and rotation angle $\beta$.

$=$

\section{Experimentation}

The methods discussed in the previous two sections were tested on a FANUC S-800 robot holding a hemispherical shaped glass bowl containing water. The bowl was instrumented with two capacitive sensors equally spaced at 180 degrees around the circumference of the bowl as shown in Figure 7. The sensors are based on a unique capacitive sensing technology for noncontact sensing of objects [8], and is used to measure the liquid's oscillation in a nonconductive container.

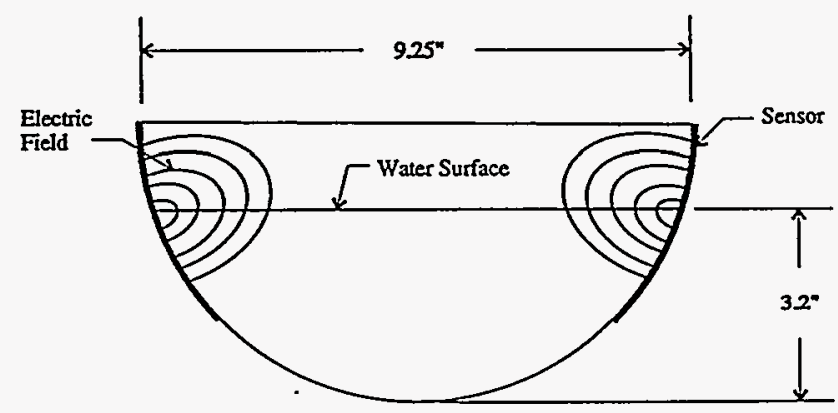

Figure 7: Hemispherical container instrumented for liquid oscillation detection. 
System identification was performed to verify the fundamental mode of oscillation predicted by the BEM. The water was caused to oscillate in the container by performing a short linear horizontal move. The container was accelerated with a triangular-shaped acceleration to a constant velocity for a total displacement of $252 \mathrm{~mm}$. Data collection was initiated at 1 second before container motion. Each sensor was sampled at $500 \mathrm{~Hz}$ for a total time of 30 seconds (15000 samples).

Figure 8 shows the output of a sensor during and following container motion. Linear calibration factors have been applied such that the magnitudes are represented in degrees with respect to the container surface. The initial sensor output of zero (with some noise) occurs before the container is in motion. The initial nonzero sensor response is due to the induced water oscillation during container motion. The remaining sensor response shows the water oscillation once the container has been decelerated to rest. Based on this data the estimated natural frequency and damping ratio were $1.67 \mathrm{~Hz}$ and 0.0093 , respectively. The BEM predicted a natural frequency of $1.68 \mathrm{~Hz}$ and a damping ratio of 0.212 . This error in damping can be reduced by adjusting the Raleigh damping coefficient according to experimental data.

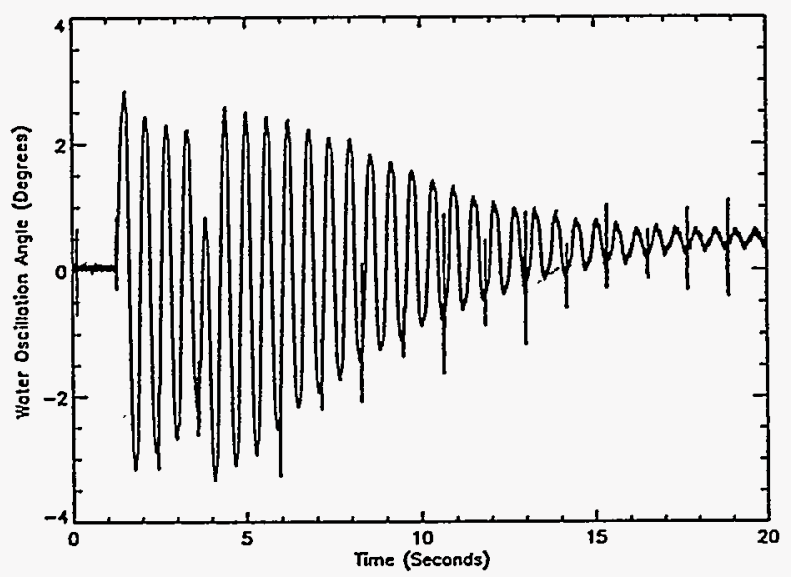

Figure 8: Measured oscillation of water in a hemispherical shaped container.

Next, the motions described in the previous two sections were tested. The horizontal motion using the IIR filter and the rotating slosh-free motion resulted in the responses shown in
Figures 9 and 10. Notice that the responses in Figure 9 are very close to that shown in Figure 4, thus validating the pendulum model and IR filter control. The periodic noise spikes in the data were the result of the sensor electronics and not a physical phenomenon in the liquid.

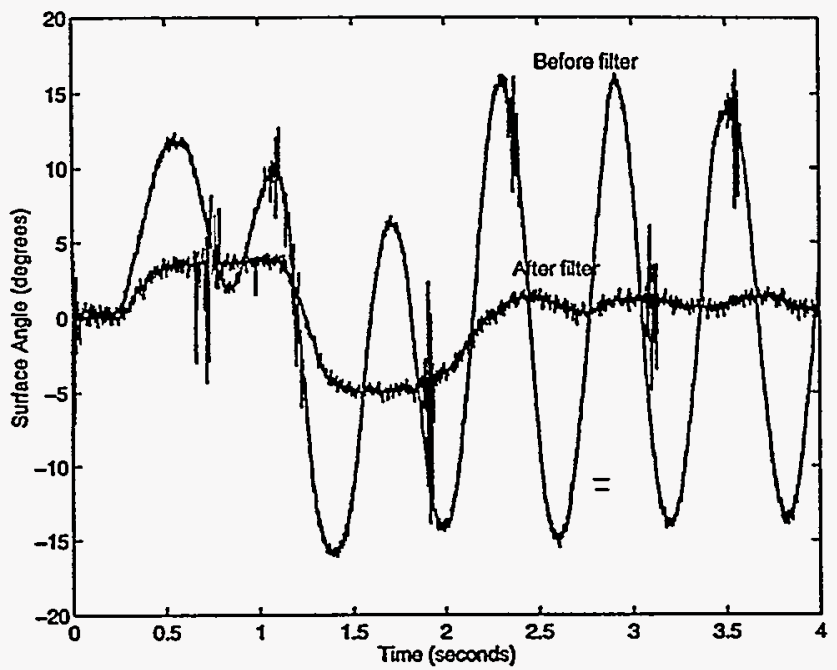

Figure 9. The measured angle $\theta$ of the fluid relative to the container for horizontal motion only.

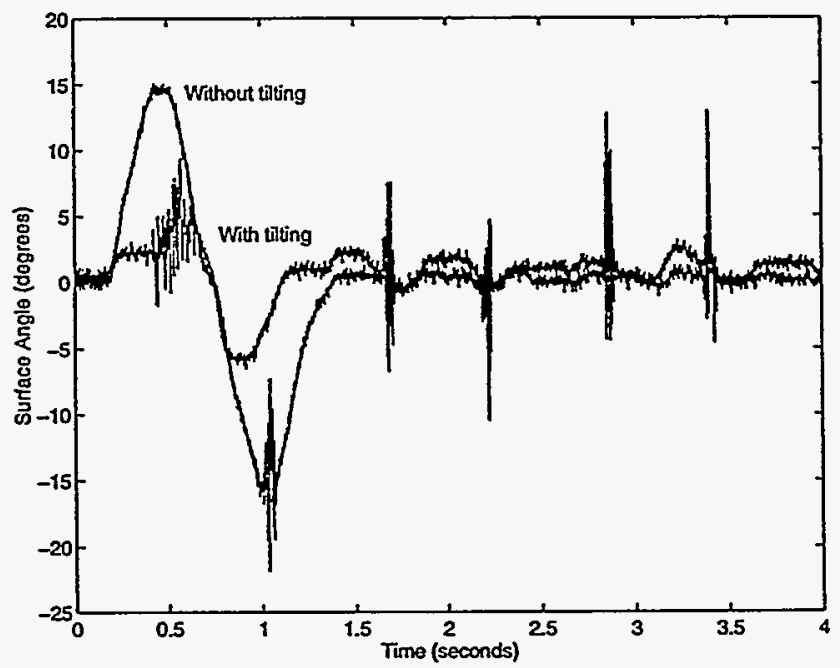

Figure 10. The measured angle $\theta$ of the fluid relative to the container with tilting.

The non-tilting response in Figure 10 is very similar to the response of the IIR filter in that it reduces residual oscillation after the motion is complete. This is because the acceleration time was set equal to the period of oscillation [5]. Ideally, the tilting response should have been 
zero. Unfortunately, the 5 degree wave which appeared upon deceleration was the result of an induced out of plane wave within the

hemispherical container. Since our model does not account for out of plane motion, further work is need to compensate for this effect.

\section{Conclusion}

The experimental results suggest that a simple pendulum model is accurate for describing the motion of a fluid within a moving hemispherical container. When translating the container, relatively simple control schemes can be used to control the fluid surface to remove residual oscillation after the move is complete. Rotating the container so that the fluid surface does not move relative to the container effectively removed the horizontal oscillation; however, the rapid rotation induces an unexpected out of plane oscillation. Further research is needed in three dimensional modeling of the fluid and in the appropriate control to reduce the out of plane oscillation.

\section{References}

[1] H.N. Abramson, editor, the Dynamic Behavior of Liquids in Moving Containers, NẠSA Sp-106, Washington, 1966.

[2] I.G. Currie, Fundamental Mechanics of Fluids, McGraw-Hill Co. 1974.
[3] V.J. Romero and M.S. Ingber, "A Numerical Model for 2-D Sloshing of PseudoViscous Liquids in Horizontally Accelerated Rectangular Containers," Proc. 17th International Conference on Boundary Elements, Madison, WI, July 17-19, 1995.

[4] N.C. Singer, "Residual Vibration Reduction in Computer Controlled Machines," Technical Report AI-TR 1030, MTT Artificial Intelligence Laboratory, Cambridge, MA, January 1989.

[5] B.J. Petterson, R.D. Robinett, J.C. Werner, "Parameter Scheduled Trajectory Planning for Suppression of Coupled Horizontal and Vertical Vibrations in a Flexible Rod," Proc. 1990 IEEE International Conference on Robotics and Automation, Cincinnati, May 13-18, 1990. $=$

[6] J.T. Feddema, "Digital Filter Control of Remotely Operated Flexible Robotic Structures," Proc. 1993 American Control Conference, San Francisco, California, June 2-4, 1993.

[7] A.J. Lichtenberg and M.A. Lieberman, Regular and Stochastic Motion, Applied Mathematical Sciences, Vol. 38, SpringerVerlag, New York, 1983, pp. 66-68.

[8] J.L. Novak and J.T. Feddema, "A Capacitance-Based Proximity Sensor for Whole Arm Obstacle Avoidance," Proc. 1992 IEEE Int. Conf. on Robotics and Automation, pp. 13071314, Nice, France, 1992.

\section{DISCLAIMER}

This report was prepared as an account of work sponsored by an agency of the United States Government. Neither the United States Government nor any agency thereof, nor any of their employees, makes any warranty, express or implied, or assumes any legal liability or responsibility for the accuracy, completeness, or usefulness of any information, apparatus, product, or process disclosed, or represents that its use would not infringe privately owned rights. Reference herein to any specific commercial product; process, or service by trade name, trademark, manufacturer, or otherwise does not necessarily constitute or imply its endorsement, recommendation, or favoring by the United States Government or any agency thereof. The views and opinions of authors expressed herein do not necessarily state or reflect those of the United States Government or any agency thereof. 